\title{
Ab initio structures of interacting methylene chloride molecules with comparison to the liquid phase
}

\author{
László Almásy ${ }^{\mathrm{a}, \mathrm{b}, *}$, Attila Bende ${ }^{\mathrm{c}}$ \\ a Laboratory for Neutron Scattering, Paul Scherrer Institut, $\mathrm{CH}-5232$ Villigen, Switzerland \\ ${ }^{\mathrm{b}}$ Adolphe Merkle Institute, University of Fribourg, $\mathrm{CH}-1700$ Fribourg, Switzerland \\ c Molecular and Biomolecular Physics Department, National Institute for Research and Development of Isotopic and Molecular Technologies, Donath Str. 65-103, Ro-400293, \\ Cluj-Napoca, Romania
}

\begin{abstract}
Equilibrium geometries of methylene chloride dimers have been calculated using the local version of second order Møller-Plesset perturbation theory (LMP2), combined with the density fitting (DF) technique. Five distinct energy minimum bonded conformations have been resolved. The results have been compared to the intramolecular geometries and the intermolecular orientational correlations obtained previously from neutron and X-ray scattering experiments combined with molecular dynamics and reverse Monte Carlo modeling.
\end{abstract}

\section{Introduction}

Molecular liquid methylene chloride has recently been studied by wide angle neutron and X-ray diffraction. The scattering functions were compared to molecular dynamics simulations [1] employing several model potentials, and analysed by the reverse Monte Carlo (RMC) method [2-4]. Using RMC, real space models of these liquids have been constructed in such way that the Fourier transforms of the atomic positions were in the closest possible agreement with the experimental data. From the three-dimensional models of the atomic and molecular configurations obtained by RMC and molecular dynamics, a number of characteristic quantities have been calculated, which are used to describe the structure of the liquid, including the structures of the constituent molecules. These are the partial radial distribution functions of the different nuclei, the orientational correlation functions of the molecules, and the geometries of the molecules.

The molecular geometries in [3] were obtained by an advanced version of the RMC technique [5], in which the atoms of individual molecules are not fixed within the molecule, but allowed to move in accordance to a set of geometrical constraints, which are usually the closest approach distances between the nuclei. As a result of such modeling, the molecules in the simulation box became not identical, but

* Corresponding author. Laboratory for Neutron Scattering, Paul Scherrer Institut, CH-5232 Villigen, Switzerland. Tel : +4156 310 5639; fax: +41563102939. E-mail address: laszlo.almasy@psi.ch (L. Almásy). slightly distorted with respect to their equilibrium or average structure. The distribution of the atomic positions mimics the Debye-Waller factor of thermal motions, and in general, has a symmetric, Gaussian like shape. A closely related quantity is the distribution of the bond angles, which is directly linked to the coordinates of the nuclei. The regular, belllike shape of this distribution is usually a sign of goodness of the modeling.

The structural description of a molecular liquid is not a trivial task, because, first of all, there is no well defined notion of what to be called the structure of the liquid. Instead, several characteristic functions are usually analysed, like the abovementioned distribution functions. It became apparent, that they are not always sufficient to resolve various particular features of the liquid. Recently, special functions of orientational correlations have been used for molecules of particular, tetrahedral shapes, resolving previously unknown structural features of liquid tetrachlorides [6-8]. Further analysis methods can be elaborated for other types of molecules. Most of the available experimental methods, such as scattering, can provide only averaged information, which is further smeared by instrumental effects and data analysis. Considering all these difficulties, it is clear that other experimental or theoretical tools which give more or less direct information can be helpful. One such method is the determination of the structure using first principles. Apart of the usefulness of the ab initio methods for calculating the intermolecular potentials used further in molecular dynamics simulations, the such obtained clusters containing a few molecules are sometimes reliable first guesses for the spatial correlations of the neighboring molecules in the liquid phase $[1,9-11]$. 
In the present work we performed quantum chemical calculations employing several levels of theory on individual methylene chloride molecule, as well as on energetically bonded dimers. The geometries of the obtained dimer conformations are compared with the results of the mentioned diffraction experiments.

\section{Ab initio calculations}

Equilibrium geometries for the monomer and five dimer structures of methylene chloride $\mathrm{CCl}_{2} \mathrm{H}_{2}$ have been obtained employing the local version of the second order Møller-Plesset perturbation theory (LMP2), combined with the density fitting (DF) technique [12-14] implemented in the MOLPRO program package suite [15] and using the aug-cc-pVTZ basis set. As the localization method the PipekMezey (PM) localization technique was used [16]. In order to follow the electron correlation effect in the intermolecular interaction energy, the corresponding single point energy calculations were made at DF-HF, DF-LMP2 and DF-LCCSD(T) levels of theory, using the same basis set as before. The obtained dimer conformations are displayed in Fig. 1, in decreasing order of the total energy, and some of the calculated quantities are collected in Table 1.

Four of these (A, B, C and E) are similar to those reported in Ref. [1]. Conformation $\mathrm{D}$ has been found using conformation $\mathrm{C}$ as an initial guess, but inverting the positions of the $\mathrm{H}$ and $\mathrm{Cl}$ atoms. Dimers $\mathrm{A}$ and $\mathrm{B}$ show the strongest bonding (at DF-LCCSD(T)/aug-cc-pVTZ level of calculation), with total energies of -3.58 and $-3.51 \mathrm{kcal} / \mathrm{mol}$, respectively. It is interesting to note, that these strongest conformations also show low molecular symmetries: $\mathrm{A}$ is totally asymmetric and $\mathrm{B}$ belongs to the $C_{s}$ point symmetry group, while the weaker configurations show higher symmetries ( $C$ and $D$ have $C_{2 h}$, and the E has $C_{2 v}$ symmetry).

The HF contribution is either close to zero or repulsive. The energy stability of the dimers is due to the electron correlations. These electron correlation effects are mostly given by the pair correlations (covered by the second order Møller-Plesset perturbation theory), while the high order correlations (included in the coupled-cluster theory) are small, around $0.1 \mathrm{kcal} / \mathrm{mol}$.

\section{Spatial correlation of the neighboring molecules}

The scattering experiments and simulations [1-4] provided threedimensional models of the liquid, from which all kinds of correlations can be derived. Although the in vacuo calculated contact dimers are not necessarily present in the liquid, some similarities still can be revealed between these calculated structures and the closest neighbor orientations in the liquid, obtained from the experiments.

The RMC study by Jedlovszky [2] showed, that in liquid methylene chloride, the orientational correlations extend far beyond the first coordination shells. An even longer range orientational correlation has recently been revealed for the tetrahedral $\mathrm{CX}_{4}$ molecular liquids [7], while in most of the other aprotic molecular liquids all orientational correlations vanish within the first correlation shell (Ref. in [2]). The

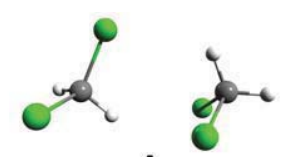

A

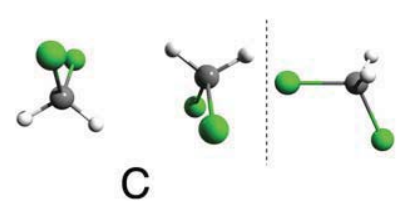

Fig. 1. Calculated dimers of methylene chloride molecules.
Table 1

Structural and energetic parameters of the optimized methylene chloride dimers.

\begin{tabular}{llclll}
\hline Dimer & $\begin{array}{l}\mathrm{d}(\mathrm{C}-\mathrm{C}) \\
(\AA)\end{array}$ & $\begin{array}{l}\mathrm{E}^{H F} \\
(\mathrm{kcal} / \mathrm{mol})\end{array}$ & $\begin{array}{l}\mathrm{E}^{\text {DF-LMP2 }} \\
(\mathrm{kcal} / \mathrm{mol})\end{array}$ & $\begin{array}{l}\mathrm{E}^{D F-L C C S D(T)} \\
(\mathrm{kcal} / \mathrm{mol})\end{array}$ & $\begin{array}{l}\mathrm{DM} \\
(\text { Debye })\end{array}$ \\
\hline $\mathrm{A}$ & 3.914 & 0.568 & -3.715 & -3.580 & 0.592 \\
$\mathrm{~B}$ & 3.851 & -0.035 & -3.639 & -3.514 & 0.791 \\
$\mathrm{C}$ & 3.650 & 1.265 & -3.342 & -3.179 & 0.039 \\
$\mathrm{D}$ & 4.094 & -0.160 & -2.806 & -2.722 & 0.010 \\
$\mathrm{E}$ & 4.280 & 0.144 & -2.549 & -2.469 & 1.462 \\
\hline
\end{tabular}

most distinct conclusion of the RMC analysis [2] was that the $\mathrm{Cl}-\mathrm{C}-\mathrm{Cl}$ planes of the nearest neighbor molecules are preferentially parallel, although no further division by the orientations with respect to the central molecule have been presented. In the theoretical structures, the C, D and E dimers show such parallel conformations. Two of them, C and $\mathrm{D}$, having antiparallel dipole moments, while the E dimer has a head-totail conformation with parallel dipole moments. It may be worth mentioning, that although all kinds of spatial correlations could in principle be extracted from the RMC or MD configurations, usually only some particular correlations are selected to be displayed and analysed, while other, eventually important or interesting correlations rest uncovered.

In a subsequent study, Bálint et al. compared the outcome of molecular dynamics simulations to the experimental X-ray and neutron structure factors [1]. They found that although the scattering experiments provide useful data for the intramolecular structure, very little information can be directly extracted about the intermolecular structure by the traditional methods of analysis. The similarity of the total scattering functions as obtained by simulations and the X-ray and neutron scattering experiments allowed them to validate the different model potentials used in the simulations. Information about the liquid structure was then extracted from the obtained MD configurations. The dominant mutual orientation of the closest neighbors in the liquid was found to be tail-to-tail, with antiparallel dipole moments. In our calculated dimers, the $\mathrm{C}$ and $\mathrm{D}$ conformations have antiparallel dipole moments, but none of them shows tail-to-tail arrangement.

In the most recent paper by Pothoczki et al. [4], the RMC analysis of the diffraction data shows that the closest contact orientations in liquid methylene chloride are preferentially of head-to-tail type. This observation, and also the absence of tail-to-tail contact dimers in ab initio calculations point to the possible inaccuracy of the given MD potentials to predict the closest neighbor arrangements.

Altogether, it can be seen that the lowest energy dimer configurations bear certain resemblance to the various features of the mutual orientations of the closest neighbors, however the most prominent, tailto-tail arrangement seen in the MD simulations is not found in the group of the five calculated dimers. Interestingly, tail-to-tail orientations have been observed in a similar molecular liquid, methylene bromide [4].

\section{Molecular geometry}

The three central bond angles have been calculated in the monomer and the five dimers. In the monomer they are: 112.32, 111.89, and $108.17^{\circ}$ for the $\mathrm{H}-\mathrm{C}-\mathrm{H}, \mathrm{Cl}-\mathrm{C}-\mathrm{Cl}$, and $\mathrm{H}-\mathrm{C}-\mathrm{Cl}$ angles, respectively. In the energetically bonded dimer conformations, these angles vary within a range of a half degree, while the angles in the monomers fall inside these intervals. That is, the intermolecular interactions with the closest neighbors cause only a slight distortion of the molecular geometry. For the various bonded dimers, these distortions change the central bond angles both in positive and negative directions. In liquid state, the many body effects can further distort the molecular geometries. It can be assumed, that energy minimization on larger clusters would lead to similar random distortions, without shifting the mean values of the central angles to one or the other side. The average value of these angles in a hypothetical frozen liquid at zero temperature should be very close 
to those in the monomer, presumably within the limits of the values calculated for the five dimers.

The intermolecular $\mathrm{C}-\mathrm{H}$ bond lengths vary by less than $0.002 \AA$ and the $\mathrm{C}-\mathrm{Cl}$ bonds by less than $0.01 \AA$, in the five studied dimers.

In the liquid state, the thermal motion and the interactions with neighbors produce a distribution of the instantaneous atomic positions, (and thus the bond angles) around their average values. The RMC modeling provides a similar distribution in the course of fitting the many thousands of atomic positions to the experimental scattering curves. In Fig. 2, the ab initio calculated values for the three central angles in the dimers are compared with the corresponding distributions of the bond angles in the liquid state, as obtained by RMC modeling.

The experimental distributions are centered approximately around the theoretically obtained bond angles, and are much broader than the variances in the angles obtained for the different dimers. Both results are trivial, as in the simulated molecules the bond angles are limited by the used geometric constraints, and the broad distribution of the angles corresponds to the thermal fluctuations in the positions of the nuclei.

Further information can be obtained by comparing the centers of the distributions. The center of the experimental (scattering plus RMC) cosine distributions has been determined by fitting Gaussian functions to the experimental distribution of the bond angles, and the results are collected in Table 2. The variances for the diffraction data are obtained as the fitting error of the center of the Gaussian function, while the error bars for the angles in the ab initio dimers are taken as the maximum deviations of the individual values from the average over the five dimers and the monomer. We can notice, that in the two experiments (scattering + RMC and the ab initio), only the mean values of the $\mathrm{H}-\mathrm{C}-\mathrm{Cl}$ angles are similar, while the differences between the experimental and theoretical values of the other two angles are beyond the combined error bars.

Assuming that the theoretical values represent the average values of the bond angles in the liquid, the observed differences can be attributed to the specifics of the diffraction experiments and their analysis. The RMC modeling is based on simultaneous fitting of the total structure factors obtained in the neutron and X-ray scattering experiments by a weighted sum of the partial structure factors of all types of atoms in the simulation box. The weights for $\mathrm{X}$-rays are the numbers of electrons on the atoms, while for neutrons these factors do not depend in a regular manner on the atomic number. The scattering intensity of X-rays is dominated by the scattering from the $\mathrm{Cl}$ atoms. The contribution of $\mathrm{C}$ atoms is weaker, while the hydrogen is practically invisible.

In the neutron scattering experiment, the hydrogen is replaced by deuterium, which has similar scattering length as the carbon, while the scattering length of the $\mathrm{Cl}$ is larger by $50 \%$ (numerical values can
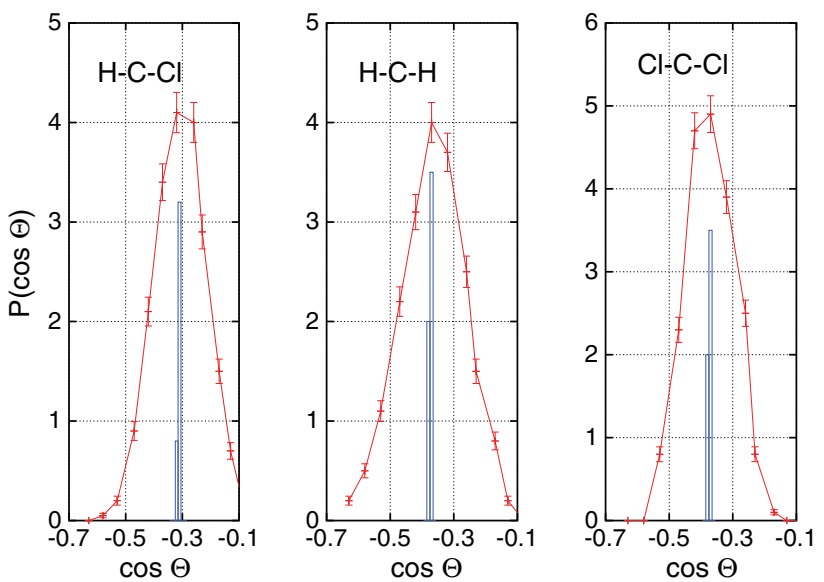

Fig. 2. Distributions of cosines of angles ligand-center-ligand, as obtained from the scattering experiment with subsequent RMC modeling (lines and points), and the same angles in the methylene chloride dimer conformations in vacuo (boxes). The heights are arbitrary.
Table 2

Intermolecular bond angles in methylene chloride as obtained ab initio, and from the simultaneous RMC fit of X-ray and neutron diffraction data.

\begin{tabular}{lll} 
& Diffraction + RMC & Ab initio \\
\hline $\cos \mathrm{H}-\mathrm{C}-\mathrm{Cl}$ & $-0.313 \pm 0.002$ & $-0.312 \pm 0.005$ \\
$\cos \mathrm{H}-\mathrm{C}-\mathrm{H}$ & $-0.366 \pm 0.001$ & $-0.373 \pm 0.005$ \\
$\cos \mathrm{Cl}-\mathrm{C}-\mathrm{Cl}$ & $-0.381 \pm 0.002$ & $-0.374 \pm 0.002$ \\
\hline
\end{tabular}

be found in Refs. [1,3]). However, the thermal motion of the proton or deuteron is much stronger, and its position is much more smeared compared to the positions of the heavier atoms.

As a result, the total scattering intensities in both experiments contain less information about the positions of the hydrogen atoms, instead, they are dominated by the partial structure factors of the intra- and intermolecular $\mathrm{Cl}-\mathrm{Cl}$ and $\mathrm{C}-\mathrm{Cl}$ pairs. The $\mathrm{RMC}$ modeling is therefore less sensitive to the positions of the hydrogen nuclei. During the simulation process the hydrogen nuclei are relatively free, even though their positions in average are still consistent with the experimental data (and with the imposed geometrical constraints). From these considerations one could expect that for methylene chloride the $\mathrm{Cl}-\mathrm{C}-\mathrm{Cl}$ angle would be measured with higher accuracy, and the $\mathrm{H}-\mathrm{C}-\mathrm{H}$ angle would be the most uncertain. The trend seen in Table 2 is just the opposite. While the equality of the $\mathrm{H}-\mathrm{C}-\mathrm{H}$ angles may be just a coincidence, the rather strong difference for the $\mathrm{Cl}-\mathrm{C}-\mathrm{Cl}$ angles indicates that the RMC produced molecular geometry does not necessarily reflect the true molecular geometry.

These results show, that in certain cases the atomic configurations obtained by RMC may have a systematic bias from the plausibly expected structure. On the other hand, these deviations are hard to detect due to the lack of appropriate tools. In the present case, the differences between the calculated and the RMC-provided intramolecular bond angles are very small compared to the magnitude of the thermal motion, therefore such small imperfections of the model configurations can hardly affect the other spatial correlations obtained by the simulation.

\section{Acknowledgments}

We thank the NIRDIMT Data Center for the provision of computer facilities. This work was supported partially by the Core-2010 Research Program of Romanian Ministry of Education, Research, Youth and Sport and by the INGRID Project No. POS-CCE-SMIS-192/2719. The financial support of the Adolphe Merkle Foundation is gratefully acknowledged.

\section{References}

[1] S. Bálint, I. Bakó, T. Grósz, T. Megyes, J. Mol. Liq. 136 (2007) 257.

[2] P. Jedlovszky, J. Chem. Phys. 107 (1997) 562

[3] Sz. Pothoczki, S. Kohara, L. Pusztai, J. Mol. Liq. 153 (2010) 112.

[4] Sz. Pothoczki, L. Temleitner, L. Pusztai, J. Chem. Phys. 132 (2010) 164511.

[5] O. Gereben, P. Jóvári, L. Temleitner, L. Pusztai, J. Optoelectron. Adv. Mater. 9 (2007) 3021

[6] R. Rey, J. Chem. Phys. 126 (2007) 164506.

[7] Sz. Pothoczki, L. Temleitner, P. Jóvári, S. Kohara, L. Pusztai, J. Chem. Phys. 130 (2009) 064503

[8] Sz. Pothoczki, L. Pusztai, J. Mol. Liq. 145 (2009) 38.

[9] T. Megyes, S. Bálint, T. Grósz, T. Radnai, I. Bakó, L. Almásy, J. Chem. Phys. 126 (2007) 164507

[10] H. Torii, J. Mol. Liq. 119 (2005) 31.

[11] A. Bende, L. Almásy, Chem. Phys. 154 (2008) 202.

[12] P. Pulay, Chem. Phys. Lett. 100 (1983) 151

[13] C. Hampel, H.-J. Werner, J. Chem. Phys. 104 (1996) 6286.

[14] M. Schütz, H.-J. Werner, J. Chem. Phys. 114 (2001) 661.

[15] MOLPRO, version 2009.1, a package of ab initio programs, H.-J. Werner, P.J. Knowles, R. Lindh, F.R. Manby, M Schütz, P Celani, T Korona, A Mitrushenkov, G. Rauhut, T.B. Adler, R. D. Amos, A. Bernhardsson, A. Berning D. L. Cooper, M.J. O. Deegan, AJ. Dobbyn, F. Eckert, E. Goll, C. Hampel, G. Hetzer, T. Hrenar, G. Knizia, C. Köppl, Y. Liu, A.W. Lloyd, R.A. Mata, A.J. May, S.J. McNicholas, W. Meyer, M. E. Mura, A. Nicklass, P. Palmieri, K. Pflüger, R. Pitzer, M. Reiher, U. Schumann, H. Stoll, A.J. Stone, R. Tarroni, T. Thorsteinsson, M. Wang, A. Wolf, Available from: http:// www.molpro.net.

[16] J. Pipek, P.G. Mezey, J. Chem. Phys. 90 (1989) 4916. 